\title{
Serviço de Atendimento Móvel de Urgência 192: Violência Contra A Mulher
}

\author{
${ }^{1}$ Mateus K Ibiapino, ${ }^{2}$ Vanessa BM Couto, ${ }^{3}$ Bernardo P Sampaio, ${ }^{4}$ Roberto AR Souza, ${ }^{5}$ Felipe A Padoin \\ ${ }^{6}$ Bárbara EB Carvalho, ${ }^{7}$ Irany S Salomão
}

\section{RESUMO}

Histórico: A violência contra a mulher constitui uma grande preocupação de saúde pública brasileira, que apresenta uma taxa de homicídio feminino que coloca o Brasil no quinto lugar no ranking mundial dessa estatística. Contudo, há poucas informações publicadas na literatura sobre mecanismos de trauma e prevalência de lesões nesse cenário. Este trabalho objetiva analisar o perfil epidemiológico das mulheres vítimas de violência através da análise de atendimentos pré-hospitalares.

Desenho do estudo: Foi realizado um estudo descritivo retrospectivo com base em coleta de dados de registros de atendimentos do Serviço de Atendimento Móvel de Urgência (SAMU) do município de Ilhéus, Bahia, realizados entre janeiro e dezembro do ano de 2014. De um universo de 1588 atendimentos a traumatizados, selecionaram-se as vítimas do sexo feminino (466), e destas aquelas cujos mecanismos de trauma foram por agressão, resultando numa amostra final de 49 atendimentos. Analisaram-se idade, hora do atendimento, dia da semana do evento, mecanismo de lesão, principal região acometida, necessidade de reposição volêmica, Revised Trauma Score (RTS) e a ocorrência de óbito pré-hospitalar. Os dados foram analisados através do software Epi Info versão 7.1.5.

Resultados: A média de idade das vítimas foi 32,6 anos. 23,4\% dos eventos ocorreram aos sábados e $21,3 \%$ aos domingos e $57,1 \%$ ocorreram no período noturno. $49 \%$ das injúrias foram causadas por espancamentos. $61,3 \%$ das lesões estavam localizadas em cabeça e pescoço. $48 \%$ das lesões eram lacerações. Utilizou-se reposição volêmica em $14,3 \%$ dos atendimentos. Apenas 1 vítima viva apresentou RTS inferior a 7,8408 . A mortalidade verificada foi de $4,1 \%$.

Conclusão: O presente estudo evidencia a preocupante realidade da violência contra a mulher, uma temática urgente e que exige uma resposta para que a sociedade brasileira e mundial alcance melhores condições de vida, segurança e igualdade.

Palavras Chave: Epidemiologia, Ferimentos e Lesões, Mulheres Agredidas, Serviços Médicos de Emergência, Saúde Pública, Traumatologia, Violência Doméstica.

\footnotetext{
${ }^{1-6}$ Estudante e Membro, ${ }^{7}$ Professor, Coordenador, e Membro Titular

${ }^{1-6}$ Colegiado de Medicina, Universidade Estadual Santa Cruz Liga Acadêmica de Trauma e Emergências LATE-UESC, Ilhéus Bahia, Brazil

${ }^{7}$ Departamento de Anatomia, Universidade Estadual Santa Cruz; Liga Acadêmica de Trauma e Emergências LATE-UESC Colégio Brasileiro de Cirurgiões e da Panamerican Trauma Society, Ilhéus, Bahia, Brazil

Corresponding Author: Mateus Kist Ibiapino, Estudante e Membro, Colegiado de Medicina, Universidade Estadual Santa Cruz; Liga Acadêmica de Trauma e Emergências LATEUESC, Ilhéus, Bahia, Brazil, Phone:+5573991137283, e-mail: mibiapino@yahoo.com.br
}

How to cite this article: Ibiapino MK, Couto VBM, Sampaio BP, Souza RAR, Padoin FA, Carvalho BEB, Salomão IS. Serviço de Atendimento Móvel de Urgência 192: Violência Contra A Mulher. Panam J Trauma Crit Care Emerg Surg 2017; 6(2):77-80.

\section{Source of support: Nil}

Conflict of interest: None

\section{ABSTRACT}

Introduction: Violence against women is a great concern in Brazilian public health, which has a female homicide rate that places the country at the fifth place of the world ranking of this statistic. However, there is little information published in literature about trauma mechanisms and prevalence of lesions in such scenario. This paper aims to study the epidemiological profile of female victims of violent trauma by analyzing prehospitalar assistance records.

Study design: A retrospective descriptive study was carried out based on the data extracted from case records of the mobile urgency medical service (SAMU, acronym in Portuguese) in the city of Ilhéus, Bahia from January to December, 2014. From a universe of 1588 cases of trauma, we selected female victims (466) and, from them, those who were subjected to aggression trauma, resulting in a final sample of 49 individuals. It was analyzed age, time of assistance, day of the week of event, lesion mechanisms, main site of injury, need of fluid replacement therapy, Revised Trauma Scores (RTS) and pre-hospitalar death. Statistical analysis was performed using Epi Info 7.1.5 software.

Results: The mean age of victims was 32.6 years. About $23.4 \%$ of events happened on Saturdays, $21.3 \%$ on Sundays, and $57.1 \%$ during the night. Nearly $49 \%$ of injuries were caused by beating. Approximately $61.3 \%$ of lesions were located on the head and neck and $48 \%$ of lesions were lacerations. Fluid therapy was used in $14.3 \%$ of times. Only a single living victim had RTS lower than 7.8408 . The mortality rate verified was $4.1 \%$.

Conclusion: This study highlights the worrying reality of violence against women, an urgent issue that requires a response so that Brazilian and world society can achieve better conditions of life, security and equality.

Keywords: Battered women, Domestic violence, Epidemiology, Prehospital care, Public health, Trauma, Wounds and injury.

\section{INTRODUÇÃO}

Segundo a Organização Mundial da Saúde, estima-se que cerca de um terço da população feminina mundial já sofreu violência doméstica. ${ }^{1}$ No Brasil, dados de 2013 indicam uma taxa de homicídio feminino de 4,8 casos a cada 100.000 habitantes, fazendo do país o $5^{\circ}$ colocado 
no ranking mundial dessa lamentável estatística. ${ }^{2}$ Essa realidade se manifesta nos mais diferentes cenários, os quais incluem agressão física para realizar roubos ou outros crimes contra patrimônio, estupros, assassinatos, violência racial, mutilação genital, prostituição forçada ou por reação à opção sexual da mulher. ${ }^{3}$ Muitas vezes, os agentes que praticam esses atos são pessoas do círculo social próximo da vítima, tais como conhecidos, familiares e cônjuges, não sendo raro, entretanto, o envolvimento de indivíduos desconhecidos ou mesmo agentes do Estado. ${ }^{3-5}$

$\mathrm{Na}$ tentativa de reverter esses indicadores, criaram-se muitos aparelhos governamentais e entidades da sociedade civil que buscam prover suporte a essas pessoas. $3,6,7$ No âmbito da saúde, também se começou a organizar ações e serviços para atender melhor à mulher vítima de abuso físico-sexual, especialmente nas questões relacionadas à transmissão de doenças sexualmente transmissíveis ou na prevenção da gravidez indesejada, quando fosse o caso. Todavia, são escassas as formulações teóricas formais que fundamentem o atendimento a essa população específica em termos de cenários de trauma e mecanismo de lesões. ${ }^{3-7}$

Tal como em outras populações vítimas de trauma, o atendimento à mulher que sofreu violência necessita de um fundamento teórico-epidemiológico para alcançar maior qualidade. A fim de contribuir com o preenchimento dessa lacuna de conhecimento populacional, o presente estudo objetivou determinar o perfil epidemiológico das mulheres vítimas de traumas violentos atendidas pelo Serviço de Atendimento Móvel de Urgência (SAMU).

\section{MÉTODOS}

Trata-se de um estudo descritivo retrospectivo que utilizou informações presentes em registros de atendimentos pré-hospitalares realizados pelo SAMU na cidade de Ilhéus, Bahia, e localidades próximas, nos meses de janeiro a dezembro de 2014. De um universo de 1588 atendimentos a traumatizados, selecionaram-se as vítimas do sexo feminino (466) e, destas, aquelas cujos mecanismos de trauma foram por agressão, resultando numa amostra final de 49 atendimentos.Desses registros, extraíram-se os seguintes dados: idade, hora do atendimento, dia da semana do evento, mecanismo de lesão, principal região acometida, necessidade e quantidade de reposição volêmica, Revised Trauma Scores (RTS)e a ocorrência de óbito pré-hospitalar.

A idade foi classificada em faixas etárias de 10 anos; a hora do atendimento foi classificada em dia (06:00 às 17:59 horas) ou em noite (18:00 às 05:59 horas); os mecanismos de trauma analisados neste estudo foram lesão por arma branca, por arma de fogo ou por espancamento; as regiões corporais acometidas foram divididas em quatro segmentos: cabeça e pescoço, tórax, abdômen e membros.
Para análise estatística, foram calculados intervalos de confiança e testes de comparação de hipóteses bicaudais através do programa Epi Info ${ }^{\mathrm{tm}_{\mathrm{m}}}$ versão 7.1.5. Foi adotado um valor de alfa de 0,05.

\section{RESULTADOS}

Do total de registros de atendimentos realizados pelo SAMU no ano de 2014, 49 preencheram os critérios de inclusão no estudo.Em relação à idade, observaram-se valores mínimo e máximo de 13 e 65 anos,além de média de 32,6 anos (IC 95\%: 29 a 36, 1 anos), mediana de 32 anos e a faixa etária mais verificada foi de 31 a 40 anos.

Sobre a distribuição dos eventos durante a semana, 23,4\% (IC 95\%: 13,6 a 37,2\%) deles ocorreram ao sábados e 21,3\% (IC 95\%: 11,9 a 34,9\%) aos domingos. Em relação ao horário, observaram-se 57,1\% (IC 95\%: 42,2 a 71,2\%) dos casos no período noturno e 42,9\% (IC 95\%: 28,8 a $57,8 \%$ ) durante o dia. A diferença entre a média de idade das vítimas do período diurno e aquelado período noturno foi de 7 anos (IC 95\%: -0,05 a 14,1 anos; $\mathrm{p}=0,06$ ). Não foi detectada relação significativa entre horários do dia e período da semana em que os eventos ocorreram $(\mathrm{p}=0,38)$. A proporção dos eventos ao longo dos dias da semana pode ser visualizada no gráfico abaixo (Gráfico 1).

Entre os diferentes mecanismos de trauma, 49\% (IC 95\%: 35 a $63 \%$ ) corresponderam a espancamentos, $47 \%$ (IC 95\%: 33 a 61\%) a agressão por armas brancas e 4\% (IC 95\%: -1,5 a 9,5\%) a agressão por armas de fogo. Não foram detectadas diferenças significativas na distribuição das causas de trauma por horário do dia $(p=0,6)$ ou por período da semana $(p=0,18)$.No estudo das regiões mais acometidas, registraram-se 61,3\% (IC 95\%: 46,2 a 74,8\%) das lesões em cabeça e pescoço, 34,7\% (IC 95\%: 21,7 a $49,7 \%$ ) em membros e 2\% (IC 95\%: 0,05 a 10\%) em tórax, assim como em abdômen.As lesões mais verificadas foram lacerações (48\%; IC 95\%: 33,9 a 62,1\%), contusões

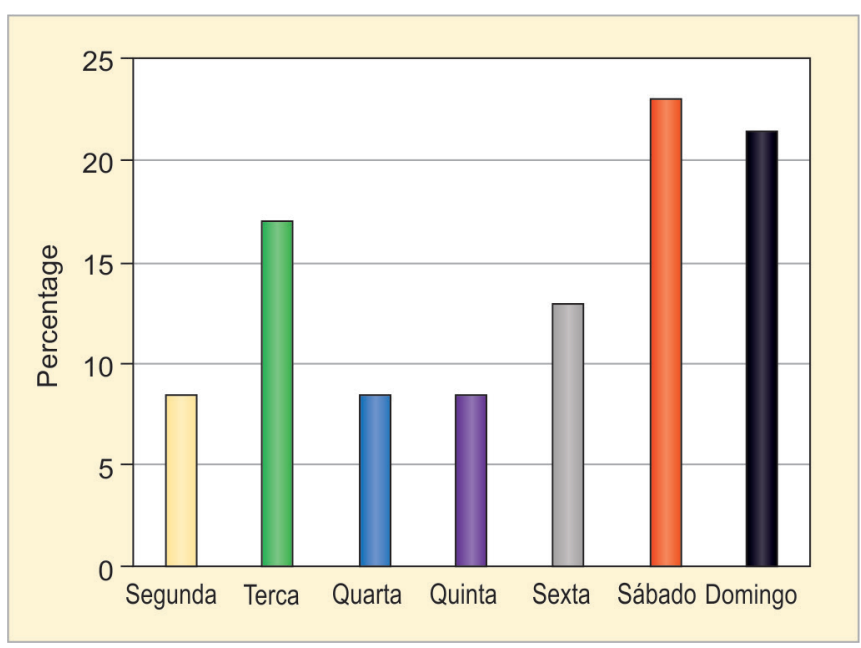

Gráfico 1: Distribuição dos traumas violentos contra mulheres através dos dias da semana 
Quadro 1: Comparação bicaudal das diferenças de necessidade de reposição volêmica entre os diferentes mecanismos de trauma. O teste de Qui-Quadrado apontou valor de $p=0,03$.

\begin{tabular}{ll}
\hline Variáveis comparadas & Valor de $P$ \\
\hline Arma branca e espancamento & $\mathrm{P}=0.4$ \\
Arma de fogo e espancamento & $\mathrm{P}=0,22$ \\
Arma branca e arma de fogo & $\mathrm{P}=0,36$ \\
\hline
\end{tabular}

(21\%; IC $95 \%: 9,5$ a 32,5\%) e ferimentos penetrantes ( $10 \%$; IC $95 \%$ : 1,5 a $18,5 \%)$.

No que diz respeito à reposição volêmica, só se observou uso de solução fisiológica em volume igual ou menor a $1500 \mathrm{ml}$. Do total de atendimentos, 14,3\% (IC 95\%: 6 a 27,2\%) necessitaram de reposição volêmica, assim como 50\% (IC 95\%: 9 a 90\%) das vítimas de agressão por armas de fogo, $21 \%$ (IC 95\%: 4,7 a 37,3\%) das lesionadas por armas brancas e em $8,3 \%$ (IC $95 \%$ : $-2,9$ a $18,9 \%$ ) dos espancamentos. A comparação das diferentes necessidades de reposição volêmica entre os diferentes mecanismos de trauma pode ser vista no quadro abaixo (Quadro 1):

Em relação aos valores de RTS verificados, 46 indivíduos apresentaram RTS igual a 7,8408 e 1 vítima de ferimento por arma branca possuía RTS igual a 5,299. Além disso, verificaram-se 2 óbitos nesse estudo, ou seja, 4,1\% (IC 95\%: 0,5 a 14\%) do total de casos. Uma foi vítima de agressão por arma branca e a outra por arma de fogo.

\section{DISCUSSÃO}

Analisando-se o perfil etário das vítimas, observou-se que a maior parte delas são jovens adultas entre 20 e 40 anos, idade até a qual a incidência desses eventos é crescente, $\mathrm{o}$ que condiz com dados de São Paulo e do Rio de Janeiro. 8,9 Todavia, no estudo de Garbin et al. ${ }^{4}$ baseado em dados de inquéritos policiais, detectou-se maior casuística na faixa etária igual ou inferior a 15 anos de idade, devido à obrigatoriedade de instauração de inquérito nesses casos, o que pode não ser realizado a depender da vontade da vítima em faixas etárias maiores.

Em relação à distribuição dos eventos durante a semana, foi evidente a maior concentração de eventos aos sábados e aos domingos em relação aos demais dias da semana, o que concorda com dados de Uberaba-MG. ${ }^{10}$ Isso pode estar relacionado ao maior consumo de bebida alcoólica por possíveis agressores nesse período e, consequentemente, maior chance de ocorrerem atos de violência. ${ }^{8,11}$

Comparando o espaçamento temporal dos eventos durante as horas do dia com os dados encontrados na literatura, houve concordância que o período noturno, entre as 18 horas e as 6 da manhã, concentra a maior parte dos relatos de violência. ${ }^{12}$ Atribui-se essa tendência,possivelmente, à maior proximidade de mulheres com parceiros, familiares ou outros potenciais agressores durante esse intervalo, assim como, maior consumo de álcool. ${ }^{3,8,11}$

A análise dos mecanismos de trauma verificados nos atendimentos evidenciou um grande predomínio de ferimentos por espancamentos e por armas brancas, acompanhando um número total proporcionalmente elevado de lesões em segmento cérvico-cefálico e em membros. O modo de distribuição desses mecanismos, em especial a relativa raridade de ferimentos por ama de fogo, é uma característica interessante desse tipo de situação, ${ }^{4,11,13} \mathrm{o}$ que muitas vezes reflete o fato da grande parte desses eventos ocorrerem em ambiente doméstico em momentos de conflitos emocionais entre os envolvidos, além da já relatada frequente intoxicação alcóolica ou por outras drogas, como crack ou cocaína. 8,12

É interessante como a reposição volêmica foi mais utilizada nas vítimas de armas de fogo em relação às demais. É fato conhecido que a lesões por arma de fogo se caracterizam por grande absorção de energia mecânica e, consequentemente, grande poder lesivo. ${ }^{13}$ Também é importante mencionar que novas evidências sugerem que o uso intempestivo de reposição volêmica pode contribuir para pior prognóstico das vítimas de trauma, preferindo-se manter o paciente em hipotensão permissiva em situações onde os recursos materiais, técnicos e a gravidade do trauma o permitam. ${ }^{14,15}$

Neste estudo, a grande maioria das vítimas apresentou um RTS de 7,8408, com expectativa de sobrevivência acima de $98 \%$, e a vítima com RTS igual a 5,299 apresentava mais de $80 \%$ de chance de sobrevivência de acordo com Champion. ${ }^{16}$ Não conseguimos localizar dados da mesma natureza na literatura a fim de comparação, o que já evidencia a dificuldade em localizar informações especialmente direcionadas à assistência ao trauma nesta população.

No ano de 2014, registraram-se 2 óbitos ao atendimento pré-hospitalar nessa amostra de vítimas. Não foi possível o seguimento dessas pacientes, o que pode subestimar a mortalidade desses eventos. Dados do DATASUS/TABNET de 2014 apontam 32 óbitos de mulheres por trauma violento na cidade de Ilhéus somando informações de todos os serviços da rede, um número relativamente alto quando comparado a Salvador, que registrou 83 fatalidades e cuja população, naquele ano, era aproximadamente 16 vezes maior. ${ }^{17,18}$ Apesar da proporção verificada nesse estudo pré-hospitalar estar em consonância com outros dados da literatura sobre o trauma na população geral, ${ }_{1}^{10}$ a mortalidade feminina ainda é menor que a masculina quando se consideram traumas violentos, ${ }^{18}$ evidenciando que o contexto do trauma violento feminino (domicílio, discussões com 
parceiros, familiares ou conhecidos) ${ }^{4-7}$ é geralmente diferente do contexto de violência contra os homens, onde predominam as lesões por envolvimento em atos criminosos e tentativas de homicídio. ${ }^{19}$

Apesar de caracterizar-se como um problema de saúde pública relevante e disseminado, a violência contra a mulher apenas ganhou maior notoriedade no Brasil com a criação da Lei 11.340/2006 (Lei Maria da Penha). ${ }^{20}$ Ao observar-se o contexto das vítimas, percebe-se a vergonha, o medo e o desconhecimento do amparo legal que denota uma magnitude invisível da violência contra a mulher, uma vez que que ocorre principalmente no âmbito privado e é, em grande parte, perpetrada por familiares e conhecidos. ${ }^{3-5,21,22}$

Por estas características, grande parte das ocorrências são subnotificadas, contribuindo para reforçar a invisibilidade da violência contra a mulher. Lamentavelmente, este tipo de violência ganha visibilidade apenas quando ocorrem casos de grande impacto nas redes sociais e repercussão midiática, contribuindo para libertar inúmeras vítimas até então prisioneiras, encarceradas em um sofrimento solitário. ${ }^{22}$

Concluindo, este trabalho evidencia a preocupante realidade da mulher envolvida no cenário de trauma por violência. Trata-se de uma temática urgente e que exige uma resposta efetiva para que a sociedade brasileira e mundial alcancem melhores condições de vida, segurança e igualdade, especialmente em regiões distantes dos grandes centros urbanos e pólos de desenvolvimento técnico-científico e cultural.

\section{REFERÊNCIAS}

1. Sminkey L, Chaib F, Khadka S, Bannerjee P. New study highlights need to scale up violence prevention efforts globally. 2014. Available from: http://wwwwhoint/mediacentre/ news/releases/2014/violence-prevention/en/.Acessoem 10/08/2016.

2. Waiselfiz, JJ; Cebela/Flacso. Mapa da Violência 2015-Homicídio de mulheres no Brasil. 2015. Brasília: Secretaria de Políticas para as Mulheres, ONU Mulheres, Organização PanAmericana da Saúde/Organização Mundial da Saúde.

3. Schraiber, LB, d'Oliveira AF, França I Jr, Pinho AA. Violência contra a mulher: estudo em uma unidade de atenção primária à saúde. Rev. Saude Publica 2002; 36(4):470-477.

4. Garbin, CA, Garbin AJ, Dossi AP, Dossi MO. Violência doméstica: análise das lesões em mulheres. Cad Saude Publica 2006;22(12):2567-2573.

5. Biagioni M. Violência contra a mulher, uma triste realidade [Monografia]. Araraquara: Faculdade de Ciências e Letras, Universidade Estadual Paulista; 2000

6. Fonseca, DH, Ribeiro, CG, Leal NSB. Violência doméstica contra a mulher: realidades e representações sociais. Psicol Soc 2012;24(2):307-314.

7. Day VP, Telles LEB, Zoratto PH, Azambuja MRF, Machado DA, Silveira MB, Debiaggi M, Reis MG, Cardoso RG, Blank P.
Violência doméstica e suas diferentes manifestações. Rev Psiquiatr Rio Gd Sul 2003;25(1):9-21.

8. Zilberman ML, Blume SB. Violência doméstica, abuso de álcool e substâncias psicoativas. Rev Bras Psiquiatr 2005;27(2): s51-s55.

9. Schraiber LB, D'oliveira AFPL, Hanada H, Figueiredo W, Couto M, Kiss L. Violência vivida: a dor que não tem nome. Interface Comun Saúde Educ2003;7(12):41-54.

10. Chavaglia SRR, Amaral EMS, Barbosa MH, Bittar DB, Ferreira PM. Vítimas de trauma por causas externas na cidade de Uberaba-MG. O Mundo da Saúde 2008;32(1):100-106.

11. Deslandes SF, Gomes R, Silva CMFP. Caracterização dos casos de violência doméstica contra a mulher atendidos em dois hospitais públicos do Rio de Janeiro. Cad Saude Publica 2000;16(1):129-137.

12. Zaleski M, Pinsky I, Laranjeira R, Ramisetty-Mikler S, Caetano R. Violência entre parceiros íntimos e consumo de álcool. Rev Saude Publica 2010;44(1):53-59.

13. Peleg K, Rivkind A, Aharonson-Daniel L, Israeli Trauma Group. Does body armor protect from firearm injuries? J Am Coll Surg 2006;202(4):643-648.

14. Reiniger LO, Sousa RMC, Nogueira LS, Costa ALS. Víctimas de accidente de tránsito sometidas a procedimientosquirúrgicos: características y complicacionestransoperatorios. Rev esc enferm USP 2012;46:58-64.

15. Waibel, BH; Rotondo, MMF. Damage control surgery: it's evolution over the last 20 years. Rev Col Bras Cir 2012;39(4): 314-321.

16. Champion HR, Sacco WJ, Copes WS, Gann DS, Gennarelli TA, Flanagan ME. A revision of the Trauma Score. J Trauma 1989;29(5):623-629.

17. Brasil. Ministério da Saúde. DATASUS [Internet]. Informações de Saúde. Estatísticas vitais. Óbitos por causas externas: mulheres vítimas de violência em 2014. Brasília (DF): Ministério da Saúde; 2016. [citado 2016 Sept 7]. Disponível em: <http:// tabnet.datasus.gov.br/cgi/tabcgi.exe?sim/cnv/ext10ba.def>.

18. Brasil. Resolução $n^{\circ} 2$, de 26 de agosto de 2014. Divulga a estimativa da População para Estados e Municípios com data de referência em $1^{\circ}$ de Julho de 2014. Diário Oficial da União, Brasília,n. 165, 26 de Agosto de 2014, p. 99, Seção I.

19. Leal SMC, Lopes MJM. A violência como objeto da assistência em um hospital de trauma: "o olhar" da enfermagem. Ciênc Saúde Coletiva 2005;10(2):419-431.

20. Brasil. Presidência da República. Lei 11.340, de 7 de agosto de 2006. Cria mecanismos para coibir a violência doméstica e familiar contra a mulher, nos termos do $\S 8^{\circ}$ do art. 226 da Constituição Federal, da Convenção sobre a Eliminação de Todas as Formas de Discriminação contra as Mulheres e da Convenção Interamericana para Prevenir, Punir e Erradicar a Violência contra a Mulher; dispõe sobre a criação dos Juizados de Violência Doméstica e Familiar contra a Mulher; altera o Código de Processo Penal, o Código Penal e a Lei de Execução Penal; e dá outras providências. Diário Oficial da União 2006; 8 ago.

21. Silva LES, Oliveira MLC. Violência contra a mulher: revisão sistemáticada produção científica nacional no período de 2009 a 2013. Ciência \& Saúde Coletiva 2015;20(11):3523-3532.

22. Garcia, LP. A magnitude invisível da violência contra a mulher. Epidemiol ServSaude 2016;25(3):451-454. 